\title{
Annals of Breast Cancer
}

Open Access | Research Article

\section{Long-term outcome data for patients with HER2- positive early-stage breast cancer treated with adjuvant trastuzumab: Benefit outside clinical trial setting}

\author{
Quintyne $\mathrm{KI}^{1,2^{*}}$; Woulfe $\mathrm{B}^{3}$; Coffey JC ${ }^{2,4}$; Gupta $\mathrm{RK}^{2,3}$ \\ ${ }^{1}$ Department of Public Health, HSE North-East, Ireland \\ ${ }^{2}$ Graduate Entry Medical School, University of Limerick, Ireland \\ ${ }^{3}$ Department of Medical Oncology, University Hospital Limerick (UHL), Ireland \\ ${ }^{4}$ Department of Surgery, UHL, Ireland
}

\section{*Corresponding Author(s): Quintyne KI}

Department of Public Health, Graduate Entry Medical

School, University of Limerick, HSE North-East, Meath, Limerick, Ireland

Email: Keithl.Quintyne@hse.ie

Received: Mar 21, 2018

Accepted: May 28, 2018

Published Online: June 07, 2018

Journal: Annals of Breast Cancer

Publisher: MedDocs Publishers LLC

Online edition: http://meddocsonline.org/

Copyright: (C) Quintyne KI (2018). This Article is distributed under the terms of Creative Commons Attribution 4.0 International License

Abbreviations: DFS: Disease-Free survival; EBC: Early-stage breast cancer; ER: Oestrogen receptor; HER2: Human epidermal growth-factor receptor 2; IQR: Interquartile range; LN: Lymph node; MDT: Multi-disciplinary team; OS: Overall survival; PgR: Progesterone receptor; REC: Research ethics committee; SLNB: Sentinel lymph node biopsy; UHL: University hospital limerick

\section{Abstract}

Background: Adjuvant treatment options for HER2positive Early-Stage Breast Cancer (EBC) have grown in recent years. The addition of adjuvant trastuzumab therapy for one-year to standard chemotherapy has been shown in several Randomized Controlled Trials (RCTs) to improve Disease-Free Survival (DFS) and Overall Survival (OS) in patients with high-risk HER2-positive EBC. This study aimed to review the long-term outcome data for patients with HER2positive EBC who were treated with adjuvant trastuzumab therapy in a designated cancer centre.

Methods: Data included all women diagnosed with HER2positive EBC between $1^{\text {st }}$ January 2001 and $31^{\text {st }}$ January 2010 ( $N=147)$. Retrospective evaluation of healthcare records for clinical, demographic, and pathologic data was undertaken. Most had adjuvant trastuzumab following systemic chemotherapy (80/147; 54.4\%). Kaplan-Meier estimates were used to evaluate whether one-year trastuzumab administration was associated with improved DFS and OS. Additionally, cohorts were generated by pathologic tumour size and lymph node involvement to stratify outcome measures (i.e. DFS and OS) by risk features.

Results: Median follow-up was 59.8 months. Patients with HER2-positive EBC who received adjuvant trastuzumab demonstrated improved 5-year DFS (75\% to 91\%; $p=$ $0.024)$, and trend for improved OS (74\% to $80 \% ; p=0.103)$. Kaplan-Meier estimates stratified by pathologic tumour size based on receipt of trastuzumab revealed that patients with tumours $\geq 2.01 \mathrm{~cm}$ had improved 5-year DFS (66\% to $88 \%$; $p=0.031)$, and 5 -year OS (66\% to $79 \% ; p=0.134)$. While

Cite this article: Quintyne KI, Woulfe B, Coffey JC, Gupta RK. Long-term outcome data for patients with HER2-positive early-stage breast cancer treated with adjuvant trastuzumab: Benefit outside clinical trial setting. Ann Breast Cancer. 2018; 1: 1002. 
Kaplan-Meier estimates stratified by lymph node involvement based on receipt of trastuzumab showed that patients with node positive tumours had improved 5-year DFS (53\% to $93 \%$; $p<0.001)$, and 5-year OS $(50 \%$ to $83 \%$; $p<0.001)$.

Conclusion: This study has shown that long-term cancerspecific outcomes (i.e. DFS and not OS) were improved for patients with HER2-positive EBC who received adjuvant trastuzumab. This benefit was greater for HER2-positive EBC where (1) tumours $\geq 2.01 \mathrm{~cm}$ and/or (2) ipsilateral axillary lymph node involvement.

\section{Introduction}

Human Epidermal Growth Factor Receptor-2 (HER2), encoded by the HER2/neu proto-oncogene, is a member of a transmembrane growth factor receptor family with tyrosine kinase activity. HER2 is amplified or overexpressed in approximately $25 \%$ to $30 \%$ of tumours in patients with Female Breast Cancer (FBC), and is associated with less favourable cancer-specific outcome [1-3]. Additionally, data has also shown that patients with HER2-positive breast cancer have an enhanced benefit for use of anthracycline-based chemotherapy over non-anthracyclinebased chemotherapy [4].

Trastuzumab (Herceptin ${ }^{\circledR}$; Genentech, San Francisco, CA) is a synthetic and recombinant humanised monoclonal antibody that binds to the extracellular domain of HER2. The action of this drug consequently blocks intracellular signalling and inhibits cell proliferation in HER2-dependent tumours [3]. Trastuzumab was approved for clinical use in 1998 in tandem with chemotherapy as a first-line treatment for HER2-positive metastatic breast cancer; where it was found to improve Overall Survival (OS) more than chemotherapy alone [5]. Subsequently, the indication was extended to include use in the adjuvant setting following chemotherapy in patients with high-risk HER2-positive $\operatorname{EBC}[3,5,6]$. With this new clinical indication, it was shown to improve Disease-Free Survival (DFS) and Overall Survival (OS) when compared to chemotherapy alone for patients with HER2positive EBC.

Breast cancer research into therapeutics has developed at a rapid pace over the last few decades. Recent discoveries have afforded the delivery of individualised treatment options, and also increased long-term survival for patients with FBC. Clinical, demographic, and pathologic features (i.e. age, race, pathologic tumour size, histologic tumour type, axillary lymph node status, standardised pathologic grade, hormone-receptor status, HER2-status, p53 mutation, and lympho-vascular invasion) are well accepted as established prognostic and/or predictive factors for the selection of systemic adjuvant therapy for EBC [4].

Recent reports have explored the survival benefit afforded by adjuvant trastuzumab for high-risk patients with HER2-positive $E B C$, and some pivotal studies have reviewed long-term followup of efficacy data of adjuvant trastuzumab $[7,8]$. These all demonstrate significant cancer-specific survival benefit seen with long-term follow-up, with an acceptable safety profile including relatively low levels of cardiac adverse events. Therefore, reducing cardiotoxicity has been highlighted as a key factor of future adjuvant trastuzumab treatment strategies, particularly in the elderly and in those who would achieve little benefit from treatment [4].

This article reports the long-term follow-up data in female patients diagnosed with HER2-positive EBC (i.e. non-metastatic) that were referred and managed in a Medical Oncology Department of a designated cancer centre, and correlated outcome based on receipt of adjuvant trastuzumab following chemotherapy. The primary objective was to determine if long-term cancer-specific outcome was improved with administration of adjuvant trastuzumab. Secondary objectives of this study were to document the incidence; demographics; histologic features; and adjuvant therapies offered to patients within our region.

\section{Methods}

\section{Patient selection}

The cohort under investigation involved only female patients diagnosed with HER2-positive EBC that were referred to Medical Oncology within the period $1^{\text {st }}$ January 2001 to 31st December 2010. HER2 amplification or overexpression was assessed primarily in UHL. Any specimen with equivocal results was reviewed in a central verification laboratory in Dublin, Ireland. HER2-positive EBC was defined as:

- A result on Immunohistochemical (IHC) analysis at UHL (HercepTest, Dako Corporation) of $3+$, in a range from 0 to $3+$, or

- $\quad$ Positive result on Fluorescence In Situ (FISH) analysis of HER2 amplification (PathVysion, Abbott Molecular) for tumours that were assessed at UHL as IHC $2+$.

For the patients included in the cohort (Figure 1), the following inclusion criteria were used:

- $\quad$ Patient age at presentation $\geq 18$ years,

- $\quad$ Pathologic data available (i.e. histologic sub-type, tumour grade of differentiation, oestrogen (ER) status, progesterone (PgR) status, tumour size, number of lymph nodes harvested and positive lymph node count),

- $\quad$ Availability of records of adjuvant systemic therapies administered (i.e. trastuzumab, chemotherapy, and hormonal therapy),

- $\quad$ Availability of records of locoregional treatment (i.e. breast/axillary surgery, and radiation administered), and

- $\quad$ Patients were reviewed by the Symptomatic Breast Unit in UHL, via a combination of clinical appointments within Mid-Western Cancer Centre and General Practitioners, who returned information on recurrence and survival status.

Exclusion criteria included: patients with HER2-negative EBC and metastatic disease (both confirmed by histologic and/or radiologic examination).

\section{Data collection}

Data was derived from the Medical Oncology database, University Hospital Limerick (UHL) case records, UHL pathology records, UHL radiology reports, and UHL Breast Multi-Disciplinary Team (MDT) records.

\section{Data analysis}

Follow-up time was calculated from the date of first surgical intervention to the date of last follow-up or death. The median follow-up was 59.8 months (Interquartile Range (IQR): 37.1 to 88.9 months).

Data was entered onto an Access database (Microsoft Corp, Redmond, WA). Information was collated and exported to Excel 
(Microsoft Corp, Redmond, WA), where the data was cleaned. The final data set was anonymised (i.e. all personal identifiers were removed) and inputted into a statistical package (IBM SPSS Statistics for Mac, Version 22.0 (IBM Corp, Armonk, NY)). The cohort was analysed by applying descriptive statistics. Results from both groups (i.e. groups determined by receipt of adjuvant trastuzumab therapy) were compared to identify any differences. All variables were considered to be independent. $\chi^{2}$ test was used for categorical data.

Life table survival curves were constructed using IBM SPSS Statistics for both DFS and OS. DFS was calculated as time between the histologic diagnosis to the time of first recurrence (proven histologically and/or radiologically). OS was calculated as the time between the histologic diagnosis and time of last contact or death (from both cancer and non-cancer causes). Survival curves were estimated with the Kaplan-Meier method.

Differences identified were considered significant if $p<0.05$ (2-tailed).

The study was approved by University Hospital Limerick (UHL) Research Ethics Committee (REC).

\section{Results}

1,041 patients with EBC were referred to the Medical Oncology Department in UHL during the period under review. 660 individual histologic samples had assessable HER2 test results. The prevalence rate for patients with HER2-positive EBC in UHL cohort was $14.1 \%(147 / 1,041)$ and among tested samples $22.3 \%$ (147/660).

\section{Summary data}

A total of 147 patients with invasive HER2-positive EBC were referred to the Medical Oncology Department in UHL within the study period. Median age at presentation was 55.0 years (IQR: 45 to 62 years). Table 1 lists the demographic and adjuvant trastuzumab treatment characteristics. The majority of HER2positive EBC patients were noted to be predominantly married, mainly Caucasian, and a majority were diagnosed within the UHL region (comprising Counties Clare, Limerick, and North Tipperary).

The right-skewed age distribution is shown in Figure 2, where most of the patients were between 45 and 65 years of age.

\section{Clinical and demographic data}

Table 2 shows the clinical and demographic data for patients with HER2-positive EBC stratified by the administration of adjuvant trastuzumab. It was noted that patient's $\geq 65$ years were less likely to receive adjuvant trastuzumab (RR: $0.32 ; 95 \% \mathrm{Cl}$ : 0.14 to $0.73 ; p<0.001$ ). No other significant differences were noted between the two groups.

\section{Pathological data}

Table 3 is a summary of the pathological data for UHL cohort, stratified according to administration of trastuzumab therapy. It was shown that among patients having adjuvant trastuzumab therapy, there was greater likelihood of: lobular and mixed carcinomas (RR: $1.49 ; 95 \% \mathrm{Cl}: 0.93$ to $2.37 ; \mathrm{p}=0.094$ ); higher grade of histologic differentiation (RR: $1.30 ; 95 \% \mathrm{Cl}: 0.98$ to $1.74 ; \mathrm{p}=$ 0.074); negative PgR status (RR: $1.64 ; 95 \% \mathrm{Cl}: 1.19$ to $2.28 ; \mathrm{p}=$ 0.003 ); and lymph node metastases with N1 disease (RR: 1.97; 95\% $\mathrm{Cl}: 1.31$ to $2.96 ; \mathrm{p}=0.001$ ), N2 disease (RR: $1.79 ; 95 \% \mathrm{Cl}$ : 1.13 to $2.83 ; p=0.013$ ), and N3 disease (RR: $1.90 ; 95 \% \mathrm{Cl}: 1.22$ to $2.96 ; p=0.004)$.

\section{Adjuvant treatment data}

Table 4 is a summary of the adjuvant treatment data for UHL cohort, stratified according to administration of adjuvant trastuzumab therapy. It was shown that among patients having adjuvant trastuzumab therapy, there was more likelihood of having, adjuvant chemotherapy (RR: $6.56 ; 95 \% \mathrm{Cl}: 1.76$ to $24.49 ; \mathrm{p}$ $=0.005$ ), and adjuvant radiation therapy (RR: $1.58 ; 95 \% \mathrm{Cl}: 0.99$ to $2.52 ; \mathrm{p}=0.054)$.

\section{Survival analysis}

\section{Overall}

Figure 3 and Figure 4 show the Kaplan-Meier estimates for DFS and OS stratified based on administration of adjuvant trastuzumab therapy. There was a trend towards improved DFS ( $p$ $=0.056$ ) with adjuvant trastuzumab, while no such trend was seen for OS $(p=0.263)$. Additionally, these estimates also demonstrate that long-term benefit of adjuvant trastuzumab does not extend beyond 5-years of treatment; this was more evident in the OS plot.

Calculations for the patients with HER2-positive EBC that had adjuvant trastuzumab were revealed to have significant improvement in 5-year DFS from $75 \%$ to $91 \%$ ( $p=0.024)$, and non-significant trend for improved 5-year OS from $74 \%$ to $80 \%$ $(p=0.103)$ Table 5.

\section{Tumour size}

Figure 5 shows the Kaplan-Meier estimates for DFS and OS stratified by administration of trastuzumab therapy and also for tumour size. For tumours greater than $2.01 \mathrm{~cm}$, there was a trend for better DFS $(p=0.070)$. No other significant differences in outcome were noted.

Subgroup calculations for patients with HER2-positive EBC that had adjuvant trastuzumab were performed. Stratification by tumour size has shown that that for tumours $\geq 2.01 \mathrm{~cm}$ had a significant improvement in 5-year DFS from 66\% to $88 \%$ ( $p=$ 0.031 ), and a non-significant trend for improvement in 5-year OS from $66 \%$ to $79 \% 9(p=0.134)$ Table 6 .

Figure 6 shows the Kaplan-Meier estimates for DFS and OS stratified by administration of trastuzumab therapy and also for lymph node involvement. For patients with node-positive HER2-positive EBC, DFS $(p<0.001)$ and OS ( $p=0.001)$ were improved with administration of trastuzumab. There were no significant improvements seen for patients with node-negative HER-positive EBC.

Further subgroup calculations for patients with HER2-positive EBC that had adjuvant trastuzumab were undertaken. Stratification by lymph node involvement has shown that patients with node-positive disease had significant improvement in 5 -year DFS from $53 \%$ to $93 \%$ ( $p<0.001)$, and 5 -year OS from $50 \%$ to $83 \%(p<0.001)$ Table 7.

\section{Discussion}

Several pivotal studies have demonstrated that the addition of adjuvant trastuzumab therapy for 1 year to standard chemotherapy can significantly improve DFS and OS versus standard chemotherapy alone $[5,6,9]$. This present study reports on long-term follow-up and outcome, based on receipt of adjuvant trastuzumab amongst patients with HER2-positive EBC. 
Results from the UHL-based cohort have shown that 5-year DFS (75\% to 91\%; $p=0.024$ ) were improved with administration of adjuvant trastuzumab, with a non-statistically significant trend for 5 -year OS (74\% to $80 \% ; p=0.103)$. These findings are not consistent with published reports, where adjuvant trastuzumab conferred both DFS and OS advantage. This might be related to the non-randomised and retrospective nature of study design employed, as it included all patients with HER2-positive EBC irrespective of their eligibility to undergo biological therapy with trastuzumab.

On subgroup analysis, varied outcome was observed with administration of adjuvant trastuzumab in patients with HER2positive EBC tumours based on pathologic tumour size. In tumours $\geq 2.01 \mathrm{~cm}$ have shown that 5 -year DFS (66\% to $88 \%$; $p=$ 0.031 ) not 5 -year OS (66\% to 79\%; $p=0.134$ ) revealed improved outcome. The literature has reported differential outcome based on tumour size. Adjuvant trastuzumab has been shown to be beneficial for tumours $>1.00 \mathrm{~cm}$, however smaller studies have revealed an absolute risk reduction by 2 to $7 \%$ irrespective of tumour size $[5,10]$.

Further subgroup analysis (using log rank to compare estimates) has revealed that HER2-positive lymph node involvement had improved 5-year DFS (53\% to 93\%; $p<0.001)$ and 5 -year OS ( $50 \%$ to $83 \%$; $p<0.001$ ). This is supported by the literature demonstrating a greater benefit for patients with nodepositive HER2-positive EBC receiving trastuzumab $[5,6,11]$. In general, the DFS and OS indices produced for this cohort of patients with HER2-psoitive EBC who received adjuvant trastuzumab were lower than published results. However, the present study included "all-comers", and again due its retrospective design had a greater proportion of older patients (some with medical co-morbidities precluding them from administration of trastuzumab), as well as patient with larger tumours and axillary lymph node involvement.

The clinical and demographic data shows that the median age for UHL HER2-positive cohort was 55 years. This was greater than 49 years reported in the HERA trial, but comparable to patient cohort in PHARE trial $[5,9]$. This variation may be due to younger patients more commonly being enrolled into clinical trials. In this project, patients receiving trastuzumab had a: young median age ( 54 years versus 57 years); and lower proportion $\geq 65$ years ( $6 \%$ versus $28 \%$; $p=0.007$ ) when compared to patients not receiving adjuvant trastuzumab. All other parameters were comparable between the groups. The majority of patients were Caucasian, and this demographic finding was similar to the published reports $[5,6]$.

The pathological data for patients in our cohort receiving adjuvant trastuzumab had: higher proportion of Grade III HER2positive EBC ( $48 \%$ versus 31\%; $p=0.074$ ); higher PgR negative rates (65\% versus $39 \% ; p=0.003$ ); and higher proportions of lymph node involvement (75\% versus 44\%; $p<0.001$ ), when compared to patients not receiving adjuvant trastuzumab. These findings were not unexpected, as they are all indicative of less favourable prognosis, which would warrant the addition of trastuzumab to adjuvant systemic intervention $[3,5,6,9]$.

This data set also highlighted those patients receiving adjuvant trastuzumab had: higher proportions undertaking adjuvant chemotherapy (95\% versus 51\%; $p=0.005$ ); and higher proportions having radiation therapy ( $85 \%$ versus $64 \%$; $p=0.054$ ), when compared with patients not having adjuvant trastuzumab; again indicating a patient population with less favourable pathologic features. These findings were comparable to results in the literature $[6,9]$.

A strength of this study is that the clinical data was derived from 10 years of primary data, which was specifically assembled to review the long-term outcome of adjuvant trastuzumab in patients with HER2-postive EBC. Most reports in the literature are based on cohorts that are recruited for RCTs, which may not be representative of the patient cohort receiving trastuzumab in the clinical setting. Another strength is related to the completeness of the data collected. This is linked to the availability of all the relevant data in UHL. In addition, the number of unique patients identified for this cohort adds to the strength of the study.

The study has some limitations. The first limitation is related to the relatively small numbers within the cohort from a single specialised cancer centre unit, which was associated with nonrandomised consecutive patient selection. This was somewhat overcome by having comprehensive access to all cohort data. Another limitation is related to eligibility for treatment with trastuzumab, as not all patients with HER2-positive EBC received the drug. This is due to evidence that purports that it only be offered to patients with less favourable pathologic features (i.e. tumour size $>2 \mathrm{~cm}$, poorly differentiated histologic lesions, and associated lymph node involvement). This inherently means that patients who receive the agent have less favourable prognosis; but despite this, it has been shown that adjuvant trastuzumab administration in patients with HER2-positive EBC resulted in improved outcome. This highlights the translation of clinical trials' results into relevant the 'real world' setting, where patients are not super-selected. Additionally, those who did not receive trastuzumab might also have unknown differences (i.e. non-histologic features including and not limited to medical co-morbidities, cardiac issues) that might have impact on cancer-specific outcome.

\section{Conclusion}

This study was to determine and quantify the long-term cancer-specific outcome in patients with HER2-positive EBC, based on the administration of adjuvant trastuzumab therapy. Statistically significant improvements in DFS and not OS were shown to improve with adjuvant trastuzumab. Additionally, it was revealed that cancer-specific outcomes were improved in patients with HER2-positive EBC where (1) tumours were $\geq 2.01 \mathrm{~cm}$ and/ or (2) lymph node involvement.

\section{Clinical practice points}

- $\quad$ Adjuvant trastuzumab therapy is beneficial for patients with HER2-positive EBC

- $\quad$ Greatest benefit was seen in patients with high-risk features (i.e. tumours were $\geq 2.01 \mathrm{~cm}$ and/or lymph node involvement), and

- $\quad$ Benefits of adjuvant trastuzumab appear to be sustained.

\section{Acknowledgements}

Clinical and support staff in Cancer Services in UHL. 
Study period: 1st January 2001 to 31 st December 2010

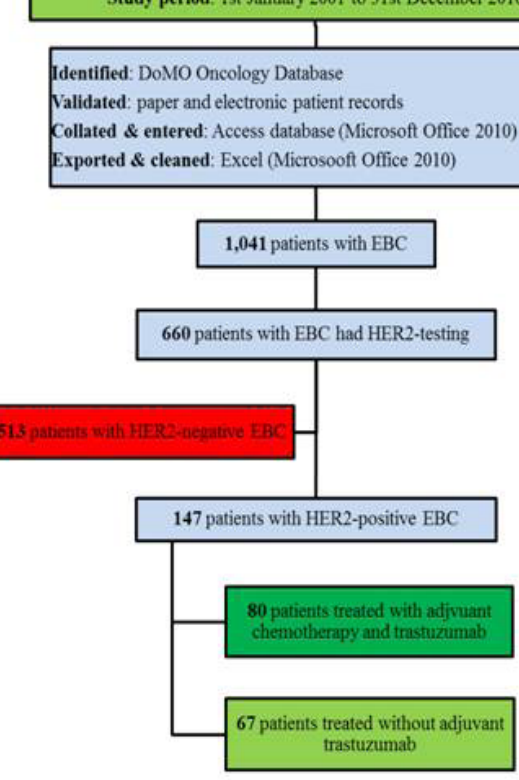

Figure 1: Flowchart describing eligible patients in UHL cohort.

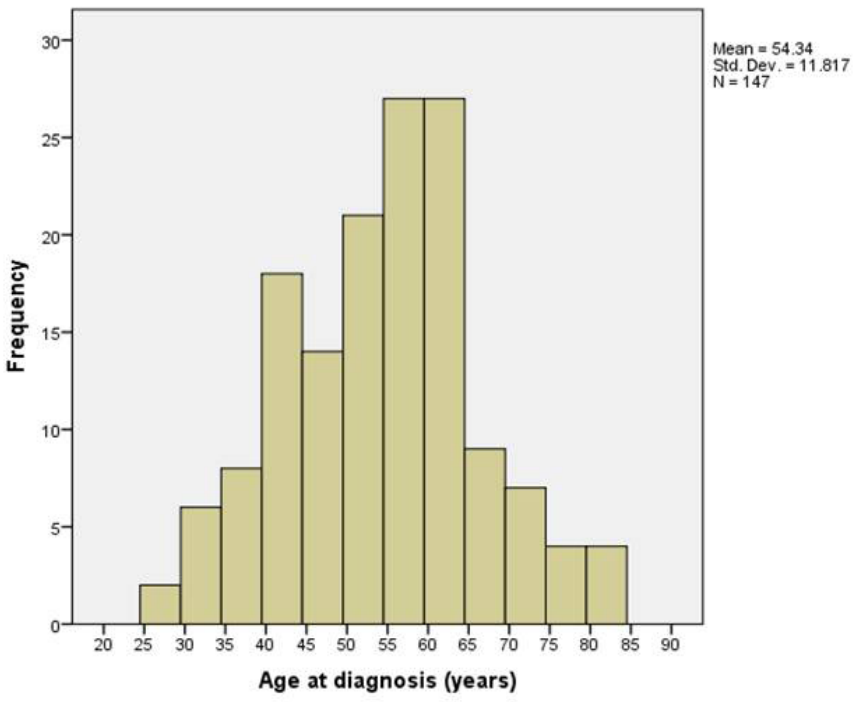

Figure 2: Age distribution for UHL HER2-positive cohort.

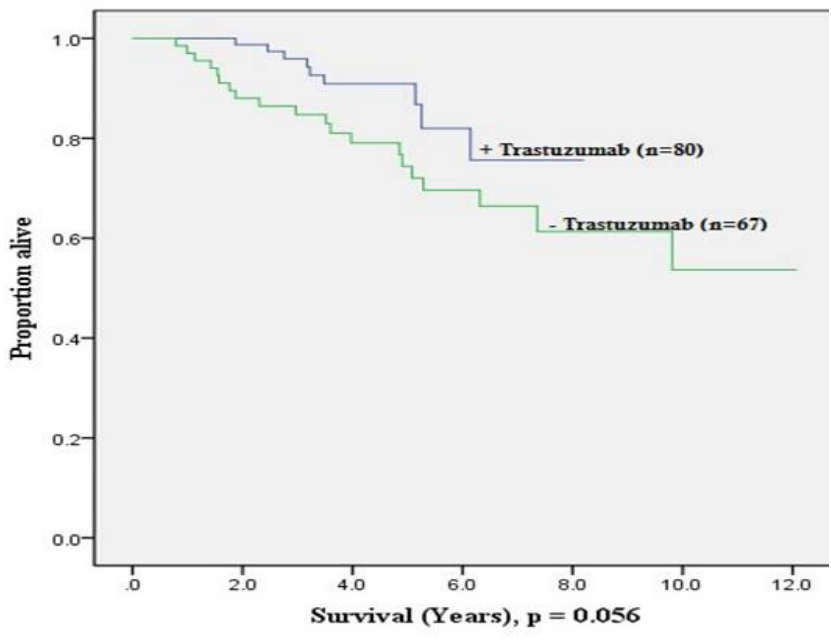

Figure 3: DFS in UHL cohort based on administration of trastuzumab.

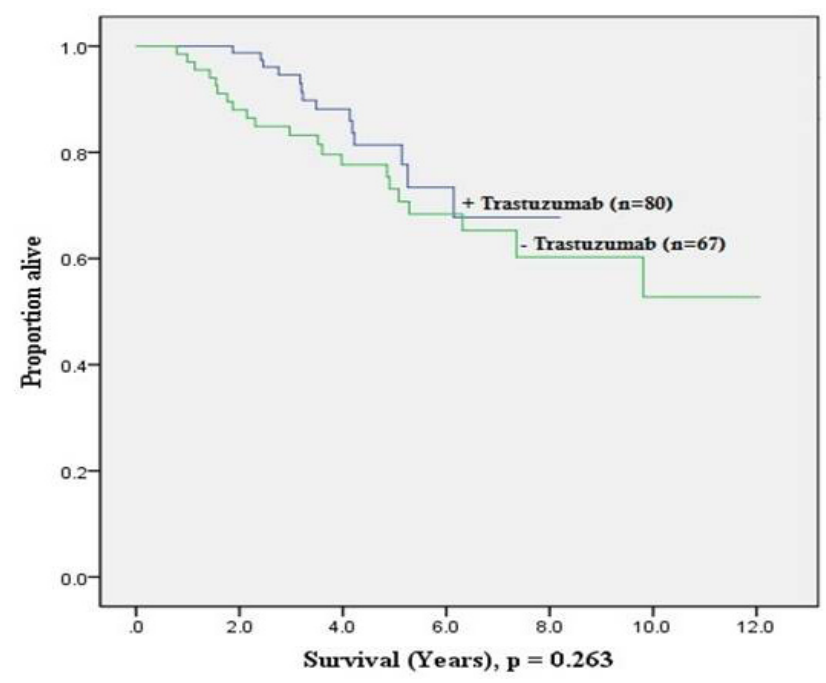

Figure 4: OS in UHL cohort based on administration of trastuzumab.
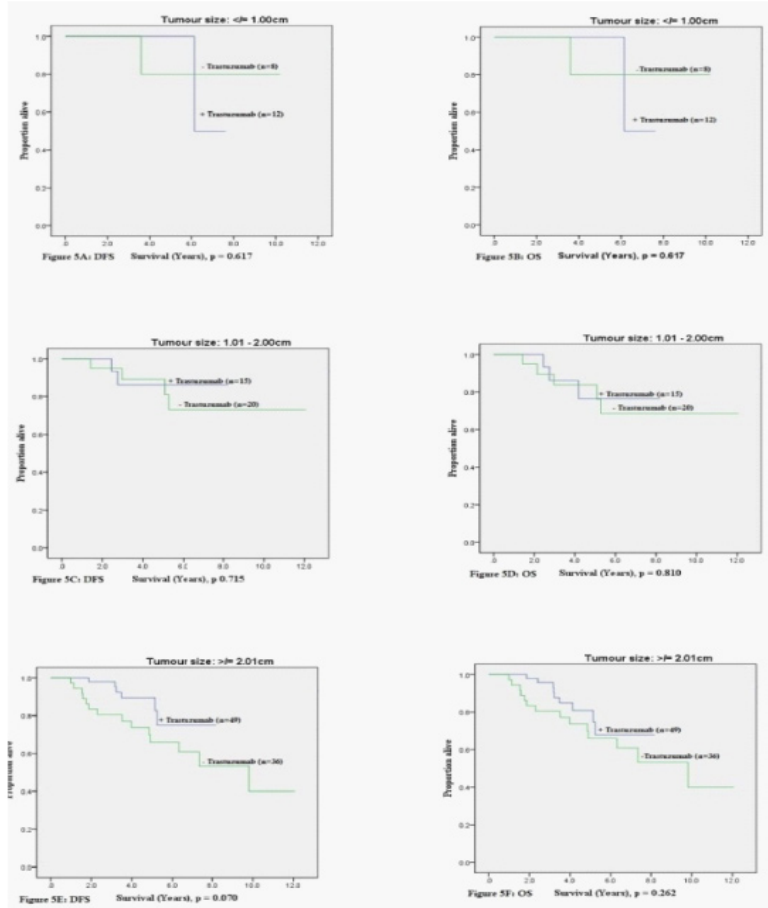

Figure 5: show DFS and OS in UHL HER2-positive cohort based on tumour size. 

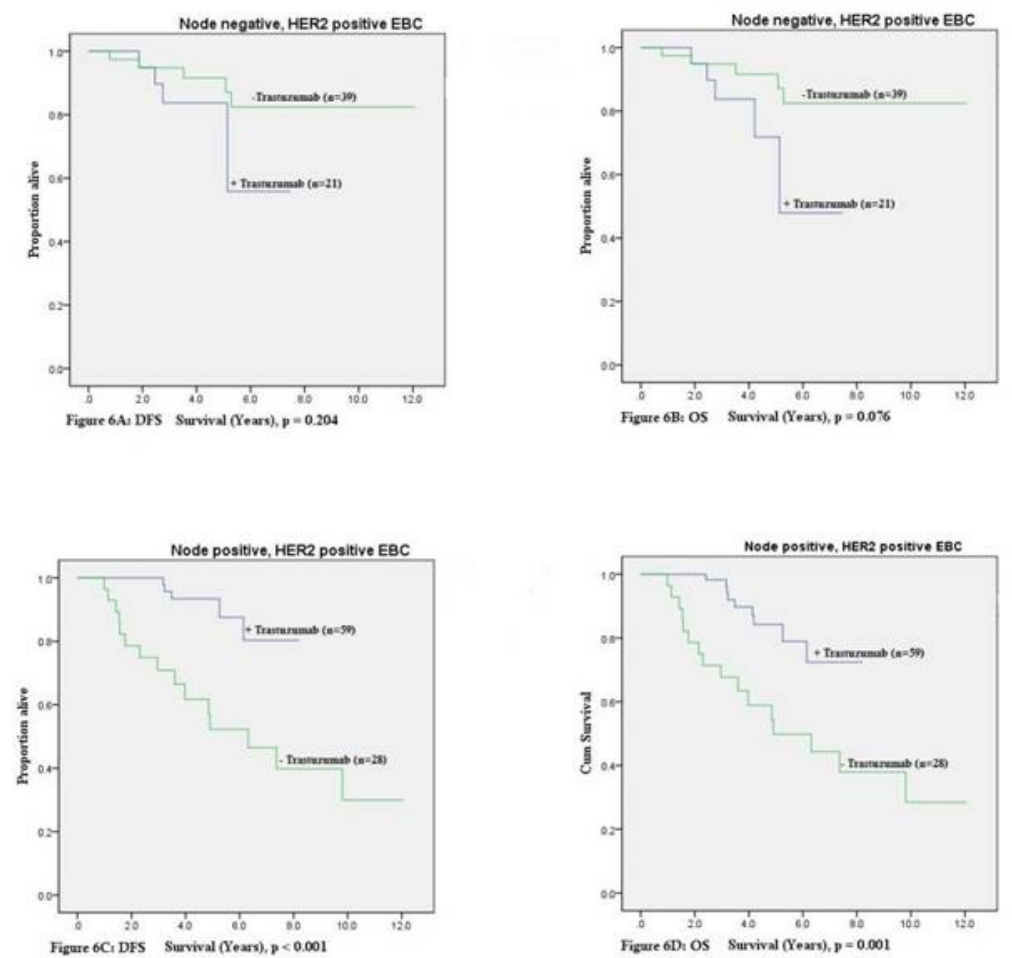

Figure 6: Show DFS and OS in UHL HER2-positive cohort based on lymph node involvement.

Table 1: Summary data for UHL HER2-positive cohort.

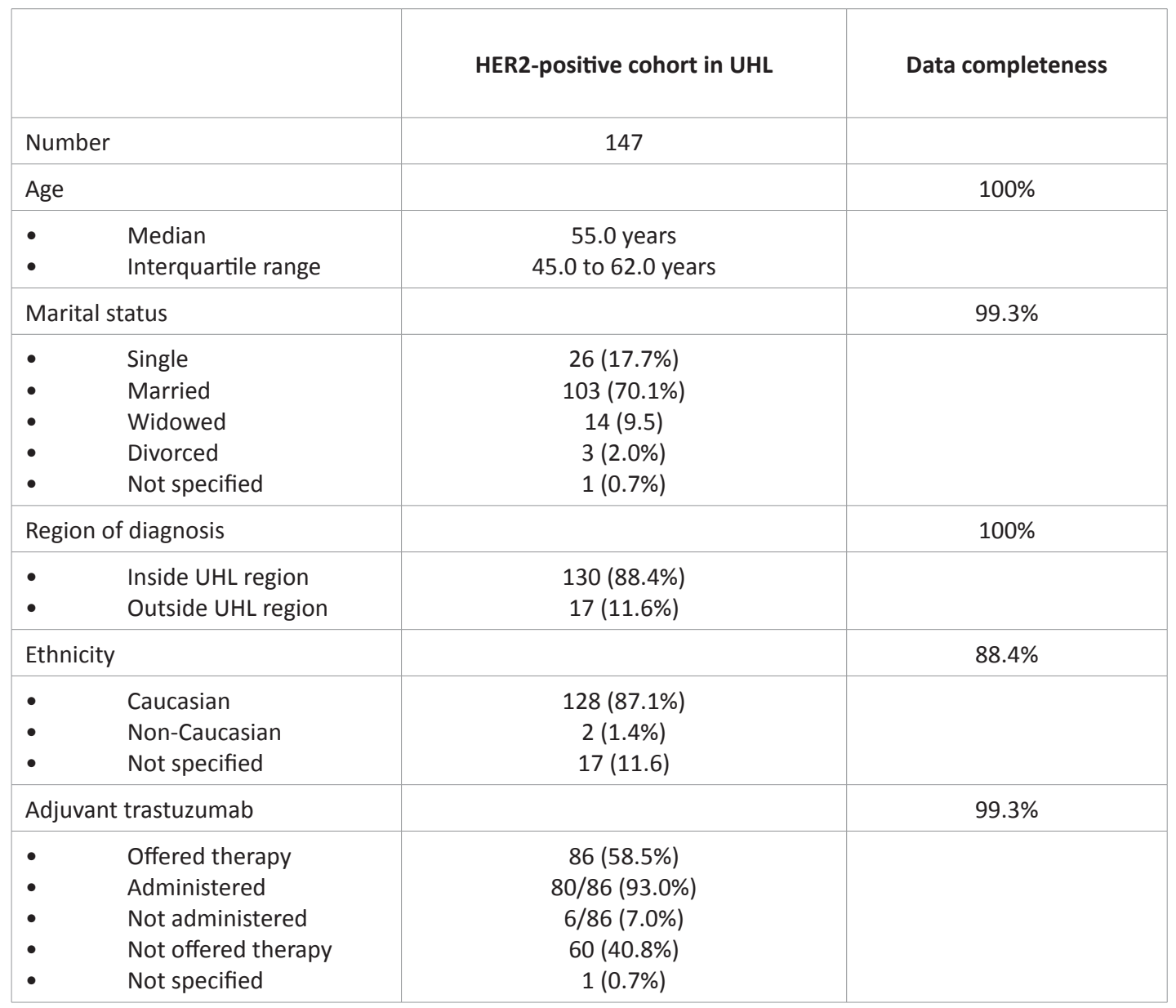


Table 2: Summary of clinical and demographic data for UHL HER2-positive cohort.

\begin{tabular}{|c|c|c|c|c|c|}
\hline & \multicolumn{2}{|c|}{ Adjuvant trastuzumab administered } & \multirow{2}{*}{ Relative risk } & \multirow{2}{*}{$\begin{array}{c}\text { 95\% Confidence } \\
\text { interval }\end{array}$} & \multirow{2}{*}{ p-value } \\
\hline & Yes & No & & & \\
\hline \multicolumn{6}{|l|}{ Age } \\
\hline - Median & 54 years & 57 years & & & \\
\hline - $\quad$ IQR (years) & 48.0 to 67.0 & 43.0 to 60.8 & & & \\
\hline - $\quad \leq 44$ years & $22(28 \%)$ & $12(18 \%)$ & Reference & & \\
\hline - $\quad 45$ to 54 years & $19(24 \%)$ & $16(24 \%)$ & 0.83 & 0.57 to 1.24 & 0.381 \\
\hline - $\quad 55$ to 64 years & $34(43 \%)$ & $20(30 \%)$ & 0.97 & 0.71 to 1.34 & 0.868 \\
\hline - $\quad \geq 65$ years & $5(6 \%)$ & $19(28 \%)$ & 0.32 & 0.14 to 0.73 & $<0.001$ \\
\hline \multicolumn{6}{|l|}{ Ethnicity } \\
\hline - Caucasian & 75 (94\%) & $53(79 \%)$ & Reference & & \\
\hline - Non-Caucasian & $0(0 \%)$ & $2(3 \%)$ & 0.28 & 0.02 to 3.59 & 0.331 \\
\hline - $\quad$ Not specified & $5(6 \%)$ & $12(18 \%)$ & 0.50 & 0.24 to 1.06 & 0.072 \\
\hline \multicolumn{6}{|c|}{ Smoking status } \\
\hline - Current & $13(16 \%)$ & $9(13 \%)$ & 1.09 & 0.72 to 1.66 & 0.669 \\
\hline - $\quad$ Former & $28(35 \%)$ & $17(25 \%)$ & 1.15 & 0.84 to 1.59 & 0.387 \\
\hline - $\quad$ Never & $34(43 \%)$ & 29 (43\%) & Reference & & \\
\hline - Not specified & $5(6 \%)$ & $12(18 \%)$ & 0.55 & 0.25 to 1.18 & 0.123 \\
\hline
\end{tabular}

${ }^{* * *}$ Percentages may not add to $100 \%$, as they have been rounded.

Table 3: Summary of pathological data for UHL HER2-positive cohort.

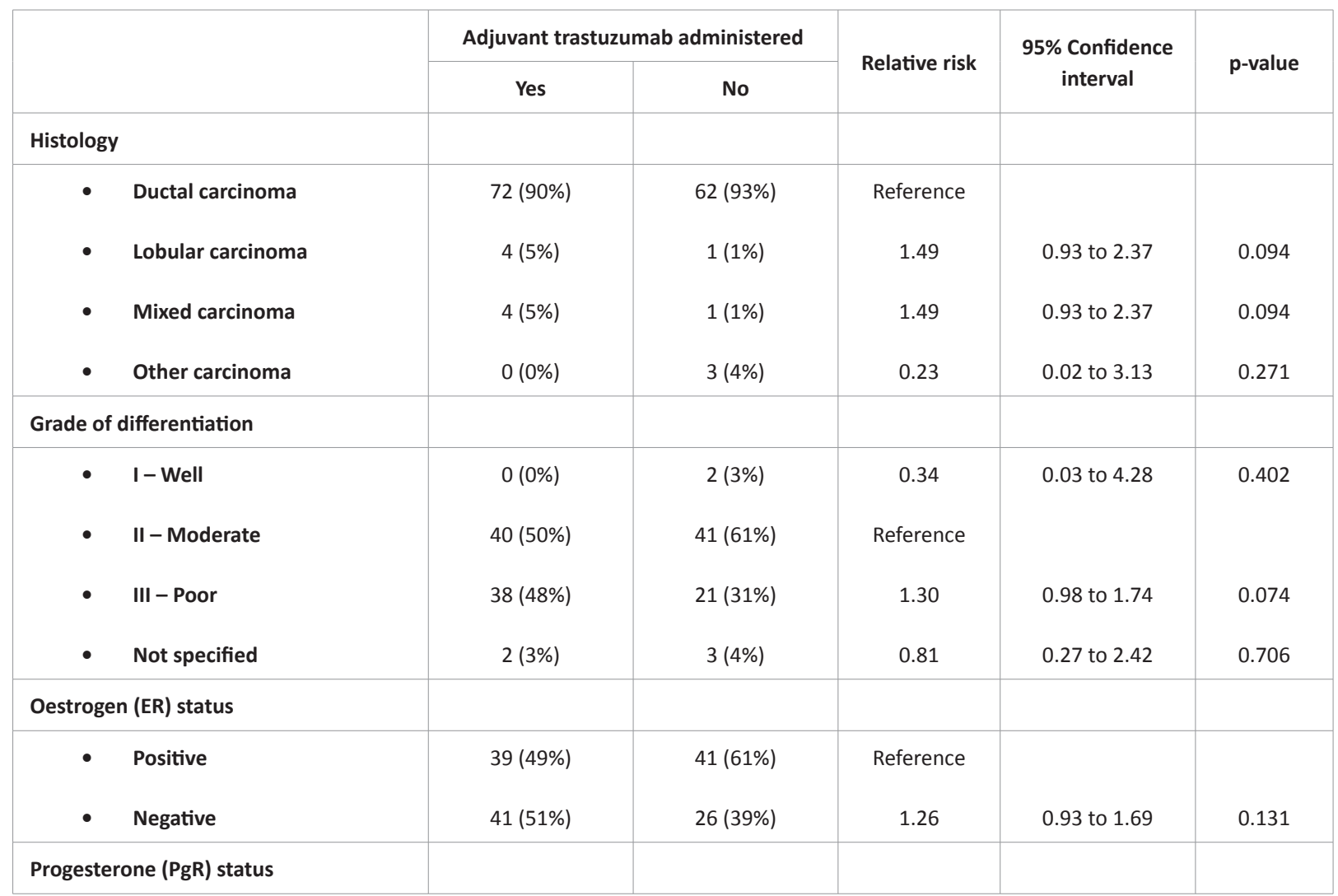




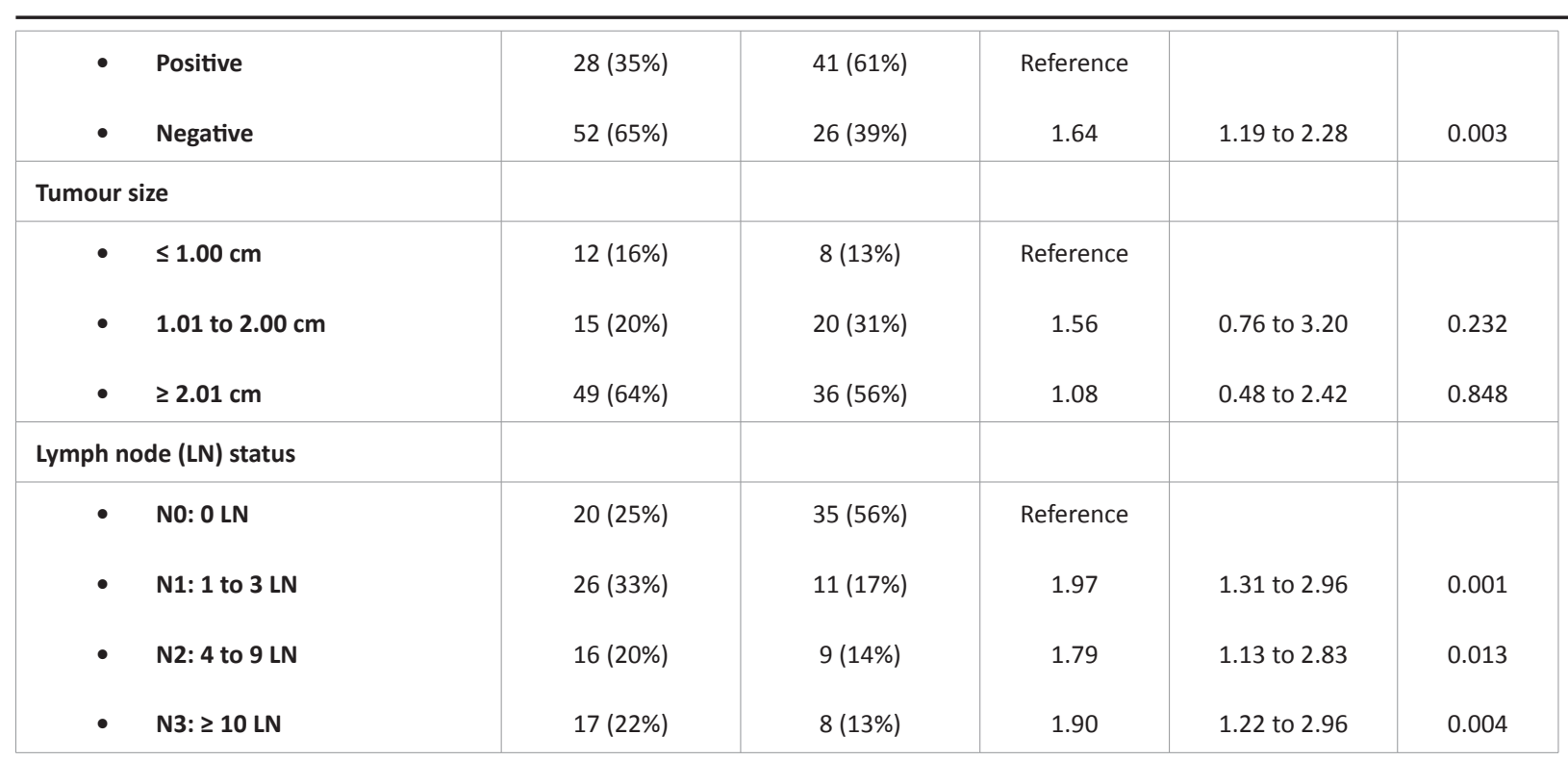

*** Percentages may not add to $100 \%$, as they have been rounded.

Table 4: Summary adjuvant treatment for UHL HER2-positive cohort.

\begin{tabular}{|c|c|c|c|c|c|c|}
\hline & & \multicolumn{2}{|c|}{ Adjuvant trastuzumab administered } & \multirow{2}{*}{ Relative risk } & \multirow{2}{*}{$\begin{array}{l}\text { 95\% Confidence } \\
\text { interval }\end{array}$} & \multirow{2}{*}{ p-value } \\
\hline & & Yes & No & & & \\
\hline \multicolumn{7}{|c|}{ Surgical intervention } \\
\hline $\begin{array}{l}\bullet \\
\bullet \\
\bullet\end{array}$ & $\begin{array}{l}\text { Breast conserving surgery } \\
\text { Mastectomy } \\
\text { No surgery }\end{array}$ & $\begin{array}{c}23(29 \%) \\
54(68 \%) \\
3(4 \%)\end{array}$ & $\begin{array}{c}23(34 \%) \\
41(61 \%) \\
3(5 \%)\end{array}$ & $\begin{array}{c}1.00 \\
1.13 \\
\text { Reference }\end{array}$ & $\begin{array}{l}0.43 \text { to } 2.34 \\
0.50 \text { to } 2.58\end{array}$ & $\begin{array}{l}1.000 \\
0.759\end{array}$ \\
\hline \multicolumn{7}{|c|}{ Axillary intervention } \\
\hline $\begin{array}{l}\bullet \\
\bullet \\
\bullet \\
\bullet\end{array}$ & $\begin{array}{l}\text { Axillary nodal clearance } \\
\text { Axillary nodal sampling } \\
\text { Sentinel lymph node biopsy } \\
\text { No surgery }^{\mathrm{b}}\end{array}$ & $\begin{array}{c}70(88 \%) \\
1(1 \%) \\
8(10 \%) \\
1(1 \%)\end{array}$ & $\begin{array}{c}54(81 \%) \\
4(6 \%) \\
6(9 \%) \\
3(5 \%)\end{array}$ & $\begin{array}{c}2.26 \\
0.80 \\
2.29 \\
\text { Reference }\end{array}$ & $\begin{array}{l}0.41 \text { to } 12.41 \\
0.07 \text { to } 9.18 \\
0.39 \text { to } 13.25\end{array}$ & $\begin{array}{l}0.349 \\
0.858 \\
0.356\end{array}$ \\
\hline \multicolumn{7}{|c|}{ Chemotherapy } \\
\hline $\begin{array}{l}\bullet \\
\bullet \\
\bullet \\
\bullet\end{array}$ & $\begin{array}{l}\text { Yes } \\
\text { No }^{c} \\
\text { Offered but declined }^{d} \\
\text { Not specified }\end{array}$ & $\begin{array}{l}76(95 \%) \\
2(3 \%) \\
2(3 \%) \\
0(0 \%)\end{array}$ & $\begin{array}{c}34(51 \%) \\
17(25 \%) \\
15(22 \%) \\
1(2 \%)\end{array}$ & $\begin{array}{c}6.56 \\
\text { Reference } \\
1.12 \\
2.00\end{array}$ & $\begin{array}{l}1.76 \text { to } 24.49 \\
0.18 \text { to } 7.09 \\
0.14 \text { to } 28.76\end{array}$ & $\begin{array}{l}0.005 \\
0.906 \\
0.610\end{array}$ \\
\hline \multicolumn{7}{|c|}{ Hormonal therapy } \\
\hline $\begin{array}{l}\bullet \\
\bullet \\
\bullet\end{array}$ & $\begin{array}{l}\text { Yes } \\
\text { No } \\
\text { Offered but declined }\end{array}$ & $\begin{array}{c}40(50 \%) \\
40(50 \%) \\
0(0 \%)\end{array}$ & $\begin{array}{c}41(61 \%) \\
25(37 \%) \\
1(2 \%)\end{array}$ & $\begin{array}{c}0.80 \\
\text { Reference } \\
0.41\end{array}$ & $\begin{array}{l}0.60 \text { to } 1.08 \\
0.04 \text { to } 4.52\end{array}$ & $\begin{array}{l}0.140 \\
0.465\end{array}$ \\
\hline \multicolumn{7}{|c|}{ Radiation therapy } \\
\hline $\begin{array}{l}\bullet \\
\bullet \\
\bullet\end{array}$ & $\begin{array}{l}\text { Yes } \\
\text { No } \\
\text { Not specified }\end{array}$ & $\begin{array}{c}68(85 \%) \\
12(15 \%) \\
0(0 \%)\end{array}$ & $\begin{array}{c}43(64 \%) \\
19(28 \%) \\
5(8 \%)\end{array}$ & $\begin{array}{c}1.58 \\
\text { Reference } \\
0.21\end{array}$ & $\begin{array}{l}0.99 \text { to } 2.52 \\
0.01 \text { to } 3.14\end{array}$ & 0.054 \\
\hline
\end{tabular}

****Percentages may not add to $100 \%$, as they have been rounded.

a:2 patients had no macroscopic disease and did not have any definitive breast surgery, and 1 patient with macroscopic disease with advanced multiple sclerosis (Available URL: http://cancer-therapy.org/CT/v8/A/4_Bahrum_et_al_10-23.pdf (Date accessed: 12th December 2016)).

b:1 patient with macroscopic disease with advanced multiple sclerosis.

c:1 patient with macroscopic disease with advanced multiple sclerosis, and 1 patient with co-morbidities that precluded chemotherapy.

$\mathrm{d}: 2$ patients who declined chemotherapy, but with high-risk HER2-positive that warranted systemic therapy. 
Table 5: Survival indices for UHL HER2-positive cohort.

\begin{tabular}{|c|c|c|}
\hline & \multicolumn{2}{|c|}{ Adjuvant trastuzumab administered } \\
\hline & Yes & No \\
\hline Disease-free survival & $N=80$ & $N=67$ \\
\hline 1-year & $100 \%$ & $97 \%$ \\
\hline 2-year & $99 \%$ & $88 \%$ \\
\hline 5-year & $91 \%$ & $75 \%$ \\
\hline 8-year & $75 \%$ & $62 \%$ \\
\hline Overall survival & $N=80$ & $N=67$ \\
\hline 1-year & $100 \%$ & $97 \%$ \\
\hline 2-year & $99 \%$ & $88 \%$ \\
\hline 5-year & $80 \%$ & $74 \%$ \\
\hline 8-year & $66 \%$ & $60 \%$ \\
\hline
\end{tabular}

Table 6: Survival indices for UHL HER2-positive cohort.

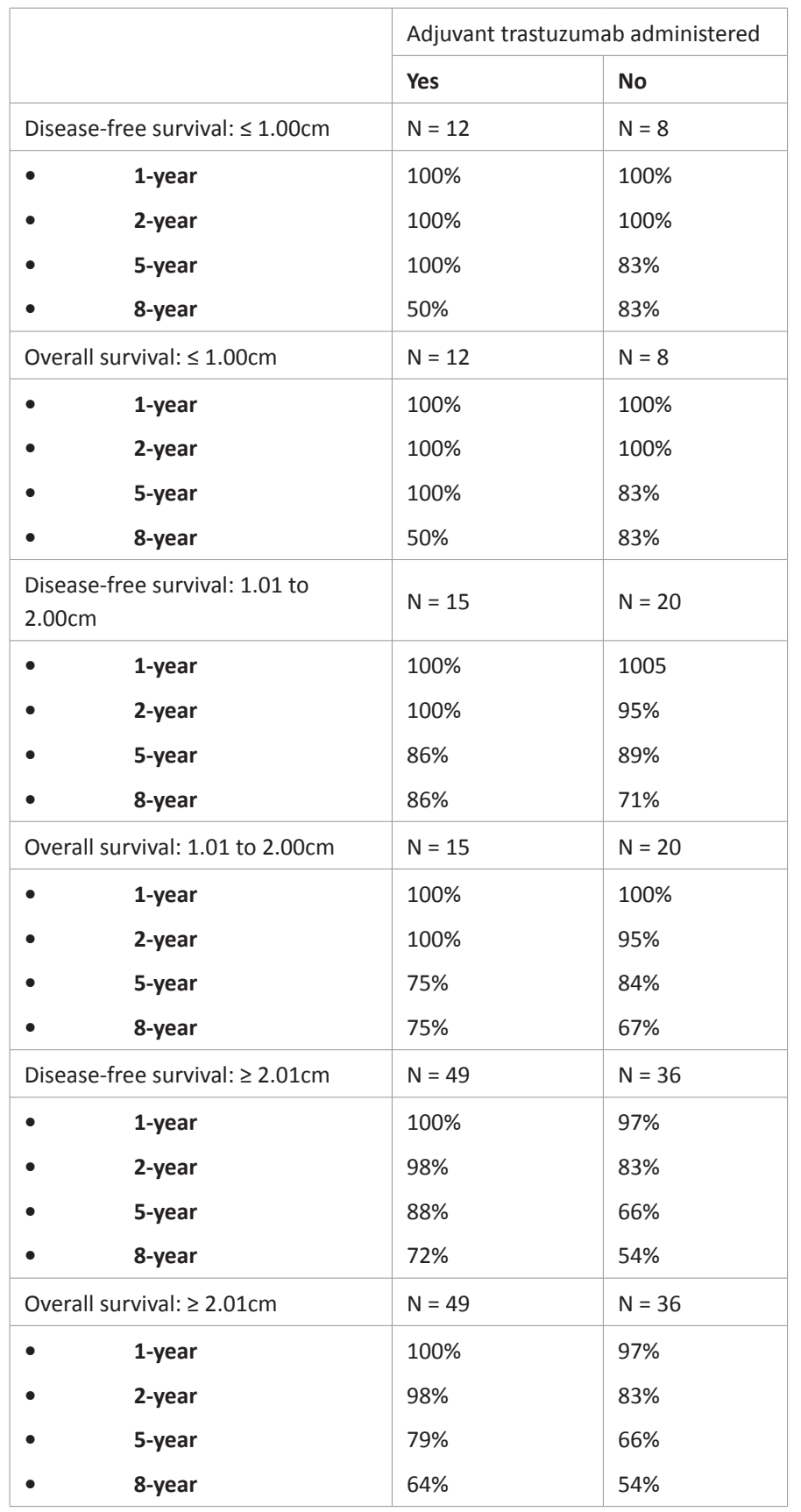

Table 7: Survival indices for UHL HER2-positive cohort stratified by lymph node involvement.

\begin{tabular}{|c|c|c|c|}
\hline & & \multicolumn{2}{|c|}{ Adjuvant trastuzumab administered } \\
\hline & & Yes & No \\
\hline \multicolumn{2}{|c|}{$\begin{array}{l}\text { Disease-free survival: node } \\
\text { negative }\end{array}$} & $N=21$ & $N=39$ \\
\hline$\bullet$ & 1-year & $100 \%$ & $97 \%$ \\
\hline$\bullet$ & 2-year & $95 \%$ & $95 \%$ \\
\hline$\bullet$ & 5-year & $84 \%$ & $92 \%$ \\
\hline$\bullet$ & 8-year & $56 \%$ & $82 \%$ \\
\hline \multicolumn{2}{|c|}{$\begin{array}{l}\text { Overall survival: node } \\
\text { negative }\end{array}$} & $N=21$ & $N=39$ \\
\hline - & 1-year & $100 \%$ & $97 \%$ \\
\hline$\bullet$ & 2-year & $95 \%$ & $95 \%$ \\
\hline$\bullet$ & 5-year & $70 \%$ & $92 \%$ \\
\hline$\bullet$ & 8-year & $47 \%$ & $82 \%$ \\
\hline \multicolumn{2}{|c|}{$\begin{array}{l}\text { Disease-free survival: node } \\
\text { positive }\end{array}$} & $N=59$ & $N=28$ \\
\hline - & 1-year & $100 \%$ & $96 \%$ \\
\hline - & 2-year & $100 \%$ & $79 \%$ \\
\hline$\bullet$ & 5-year & $93 \%$ & $53 \%$ \\
\hline - & 8-year & $80 \%$ & $40 \%$ \\
\hline \multicolumn{2}{|c|}{$\begin{array}{l}\text { Overall survival: node } \\
\text { positive }\end{array}$} & $N=59$ & $N=28$ \\
\hline$\bullet$ & 1-year & $100 \%$ & $96 \%$ \\
\hline$\bullet$ & 2-year & $100 \%$ & $79 \%$ \\
\hline - & 5-year & $83 \%$ & $50 \%$ \\
\hline - & 8-year & $71 \%$ & $38 \%$ \\
\hline
\end{tabular}

\section{References}

1. Slamon DJ, Clark GM, Wong SG, Levin WJ, Ullrich A, McGuire WL. Human breast cancer: correlation of relapse and survival with amplification of the HER-2/neu oncogene. Science (New York, NY). 1987; 235: 177-182.

2. Brufsky A. Trastuzumab-based therapy for patients with HER2positive breast cancer: from early scientific development to foundation of care. American journal of clinical oncology. 2010; 33: 186-195.

3. Slamon D, Eiermann W, Robert N, Pienkowski T, Martin M, Press $\mathrm{M}$, et al. Adjuvant trastuzumab in HER2-positive breast cancer. N Engl J Med. 2011; 365: 1273-1283.

4. Theriault RL, Carlson RW, Allred C, Anderson BO, Burstein $\mathrm{HJ}$, Edge SB, et al. Breast Cancer, Version 3.2013. Journal of the $\mathrm{Na}-$ tional Comprehensive Cancer Network: JNCCN. 2013; 11: 753761.

5. Piccart-Gebhart MJ, Procter M, Leyland-Jones B, Goldhirsch A, Untch M, Smith I, et al. Trastuzumab after Adjuvant Chemotherapy in HER2-Positive Breast Cancer. New England Journal of Medicine. 2005; 353: 1659-1672.

6. Romond EH, Perez EA, Bryant J, Suman VJ, Geyer CE, Davidson $\mathrm{NE}$, et al. Trastuzumab plus Adjuvant Chemotherapy for Operable HER2-Positive Breast Cancer. New England Journal of Medicine. 2005 ; 353: 1673-1684. 
7. Goldhirsch A, Gelber RD, Piccart-Gebhart MJ, de Azambuja E, Procter M, Suter TM, et al. 2 years versus 1 year of adjuvant trastuzumab for HER2-positive breast cancer (HERA): an openlabel, randomised controlled trial. Lancet. 2013.

8. Perez EA, Suman VJ, Davidson NE, Gralow JR, Kaufman PA, Visscher DW, et al. Sequential Versus Concurrent Trastuzumab in Adjuvant Chemotherapy for Breast Cancer. Journal of Clinical Oncology. 2011; 29: 4491-4497.

9. Pivot X, Romieu G, Debled M, Pierga JY, Kerbrat P, Bachelot T, et al. 6 months versus 12 months of adjuvant trastuzumab for patients with HER2-positive early breast cancer (PHARE): a randomised phase 3 trial. The Lancet Oncology. 2013; 14: 741-748.

10. Albanell J, Ciruelos EM, Lluch A, Munoz M, Rodriguez CA. Trastuzumab in small tumours and in elderly women. Cancer treatment reviews. 2013.

11. Araki K, Saji S, Gallas M, Pegram M, Sasaki Y. Possible available treatment option for early stage, small, node-negative, and HER2-overexpressing breast cancer. Breast cancer (Tokyo, Japan). 2012; 19: 95-103. 